\title{
BTI Impact on Logical Gates in Nano-scale CMOS Technology
}

\author{
Seyab Khan Said Hamdioui \\ Computer Engineering Laboratory \\ Delft University of Technology \\ Mekelweg 4, 2628 CD Delft,The Netherland \\ \{M.S.K.Seyab,S.Hamdioui\}@tudelft.nl
}

\author{
Halil Kukner Praveen Raghavan Francky Catthoor \\ Kapeldreef 75,B-3001 \\ Leuven, Belgium \\ \{Halil,Ragha,Francky.catthoor $\} @$ imec.be
}

\begin{abstract}
As semiconductor manufacturing has entered into the nanoscale era, Bias Temperature Instability (BTI) -Negative BTI (NBTI) in PMOS transistors and Positive BTI (PBTI) in NMOS transistors- has become one of the most serious aging mechanisms that reduces reliability of logic gates. This paper presents a simulation-based BTI analysis in both basic (such as NAND and NOR) and complex gates while considering the impact of input's duty cycle, the frequency at which they change, as well as the impact of the stressed transistor location. The simulation results show that the impact of BTI is strongly gate dependent and that in general the impact in complex gates is larger. When considering both NBTI and PBTI for basic gates, the results reveal that for a NOR gate the impact of NBTI is $2.19 \times$ higher than that of PBTI; while for a NAND gate, PBTI impact is $1.27 \times$ higher than that of NBTI. When considering different input duty cycles and their frequencies, the results show that the higher the duty cycle, the lower NBTI impact and the higher the PBTI impact regardless of the gate types and the frequency; a variation of $\pm 30 \%$ duty cycle causes a variation of up to $49 \%$ variation in the impact of NBTI and a variation of $16 \%$ in the impact of PBTI. For complex gates, the results show similar trends, but with higher impact.
\end{abstract}

Index Terms-: NBTI, PBTI, Complex gates, duty cycle, frequency, stress location

\section{INTRODUCTION}

CMOS technology miniaturization has continued unabated for more than 30 years. The miniaturization resulted in higher IC performance and density; however, it has caused reliability issues in the scaled technologies [1]. Among the reliability issues, Bias Temperature Instability (BTI) has drawn attention that degrades the performance of the MOS transistors during "ON" states at elevated temperatures [2]. The impacts of BTI on MOS transistors include threshold voltage increment and current reduction leading to performance degradation $[3,5,7]$.

BTI degradation originates from several electro-chemical sub-processes in the MOS transistors having high- $\kappa$ [3], pure silicon dioxide $\left(\mathrm{SiO}_{2}\right)$, plasma nitrided or thermally nitrided dielectric layers [8]. These sub-processes take place during the transistor "ON" state and produce charges at the $\mathrm{Si}_{-} \mathrm{SiO}_{2}$ interface [3,5]. These charges cause a transistor threshold voltage shift and consequently an additional gate delay. A unique property of BTI is annealing of the charges at the $\mathrm{Si}$ $\mathrm{SiO}_{2}$ interface during the transistor "OFF" state. The charge annealing reduces the threshold voltage shift and results in a lower additional delay. Therefore, depending on the ON/OFF ratio (which is referred to as activity factor) of the transistors, the gate will suffer from variable additional delays.

In recent years, there is an escalation of interest in BTI analysis at the gate level [10-15]. Paul et al. in [10] pioneered the work by performing NBTI analysis for the continuous inputs that resulted in the worst degradation and Haldun et al. in [11] carried out the analysis for a modified model. Kumar et al. in [12] and Khan et al. in [13] presented NBTI analysis for dynamic inputs. Luo et al. in [14] analyzed NBTI in the gates by considering stacking effect and Rakesh et al. in [15] introduced various process and design parameters in the analysis. All these analyses assume that all transistors in a gate have the same activity factor and contribute uniformly to gate delays. These approaches suffer from the very fundamental limitations as the authors have not yet investigated: (a) the organization of the transistors in a gate, (b) the interference among BTI in different transistors, (c) the workload dependency (based on activity factor e.g.) and (d) the location of the affected transistor in the gate. One recent exception for (a)-(c) is Kaczer et al. work in [21] but they only analyzed simple inverter and not extended the analysis to the other gates. Moreover, the published work focuses mainly on simple gates such as NAND, NOR, etc. However, the impact on the complex gates could be significantly different. Hence, it is vital to analyze BTI in gates that addresses all the above limitations.

This paper presents a simulation-based BTI analysis for basic and complex gates. Main contributions of the paper are:

- Incorporate both NBTI and PBTI in simulation for accurate BTI evaluation in the basic gates

- Investigate the impacts of BTI in basic gates while considering inputs with different duty cycles and frequencies. In addition, the contribution of BTI in each transistor within the gate to the overall impact is explored

- Analyze BTI in the complex gates and compare the results with those obtained for the basic gates

The rest of the paper is organized as follows: Section II presents BTI mechanisms along with a brief overview of a BTI model. Section III-A presents a model for BTI induced gate delay and a framework for BTI analysis. Section IV presents BTI analysis in the basic gates. Section V analyzes BTI in complex gates. Finally, Section VI concludes the paper. 


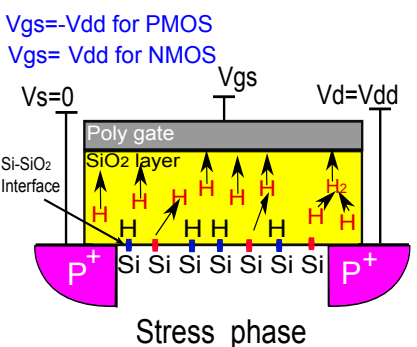

(a)
Vgs= Vdd for PMOS

Vgs=-Vdd for NMOS

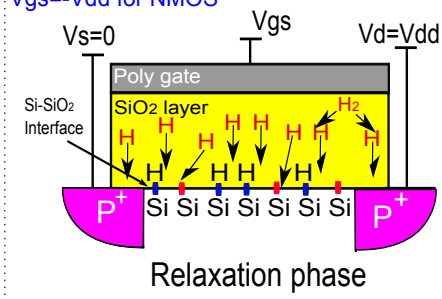

(b)
Fig. 1. Schematic view of (a) Stress phase: $\mathrm{Si}-\mathrm{H}$ bond breaking, $\mathrm{H}$ and $\mathrm{H}_{2}$ diffusions toward poly gate (b) Relaxation phase: $\mathrm{H}$ and $\mathrm{H}_{2}$ diffusions toward the $\mathrm{Si}-\mathrm{SiO}_{2}$ interface and $\equiv \mathrm{Si}$ - bond recovery

\section{BACKGROUND}

This section explains BTI mechanism that takes place inside MOS transistors. Thereafter, it reviews a well known model that relates BTI mechanism to the threshold voltage increment.

\section{A. BTI Mechanism}

BTI causes threshold voltage $\left(\mathrm{V}_{\mathrm{th}}\right)$ increments to the MOS transistors. $\mathrm{V}_{\text {th }}$ increment in a PMOS transistor that occurs under the negative gate stress is referred to as NBTI, and the one that occur in an NMOS transistor under positive gate stress is known as PBTI. Zafar et al. in [3] have carried out a comparative analysis of NBTI and PBTI impacts in MOS transistors; they concluded that either NBTI or PBTI can become more significant depending on the dielectric type.

For a MOS transistor, there are two BTI phases, i.e., the stress phase (see Fig. 1(a)) and the relaxation phase (see Fig. 1(b)). These two phases differ by the gate biasing (i.e., $V_{d d}$ or $-\mathrm{V}_{\mathrm{dd}}$ ) of the MOS transistors.

\section{Stress Phase}

In the stress phase, the Silicon Hydrogen bonds ( $\equiv \mathrm{Si}-\mathrm{H})$ breaking takes place at the Silicon-Silicon dioxide $\left(\mathrm{Si}-\mathrm{SiO}_{2}\right)$ interface as shown in Fig. 1(a). The broken Silicon bonds (三Si-) trap at the $\mathrm{Si}_{-} \mathrm{SiO}_{2}$ interface thus known as interface traps and the released $\mathrm{H}$ atoms/molecules diffuse toward the poly gate. The number of interface traps $\left(N_{I T}\right)$ depends on $\equiv \mathrm{Si}-\mathrm{H}$ bond breaking rate $\left(\mathrm{k}_{\mathrm{f}}\right), \mathrm{H}$ and $\mathrm{H}_{2}$ diffusion rates $\left(\mathrm{D}_{\mathrm{H}}\right.$ and $\left.\mathrm{D}_{\mathrm{H}_{2}}\right)$, and $\equiv \mathrm{Si}$ - bond recovery rate $\left(\mathrm{k}_{\mathrm{r}}\right)$. The overall process has been described by the reaction-diffusion (RD) model [5].

\section{Relaxation Phase}

In the relaxation phase, there is no $\equiv \mathrm{Si}-\mathrm{H}$ breaking and $\mathrm{H}$ or $\mathrm{H}_{2}$ diffusions towards the poly gate as shown in Fig. 1(b). However, $\mathrm{H}$ atoms/molecules diffuse back towards the $\mathrm{Si}-\mathrm{SiO}_{2}$ interface and anneal the $\equiv \mathrm{Si}$ - bonds. The annealing process reduces $\mathrm{N}_{\mathrm{IT}}$ at the $\mathrm{Si}-\mathrm{SiO}_{2}$ interface and reduces the degradation caused during the stress phase.

\section{B. BTI Model}

In recent years, researchers are putting more efforts to analyze BTI mechanism that takes place inside the MOS transistors [3,5,6,21]. Kaczer et al. of [6,21] have recently developed a more complete model of the transistor level of NBTI. But this model is not yet available at the time of our work, and in $[6,21]$ no higher level modeling has been proposed. Alam et al. in [5] have modeled the overall dynamics of NBTI as a Reaction Diffusion (RD) process. The model is usable at a higher level such as gate level. Because this work analyze BTI at the gate level, model of [5] will be used.

The model relates $\mathrm{N}_{\mathrm{IT}}$ generated during the stress phase with the time $(t)$ as follows:

$$
N_{I T}(t)=\left(\frac{k_{f} \cdot N_{o}}{k_{r}}\right)^{2 / 3} \cdot\left(\frac{k_{H}}{k_{H_{2}}}\right)^{1 / 3} \cdot\left(6 . D_{H_{2}} \cdot t\right)^{1 / 6},
$$

where $\mathrm{N}_{\mathrm{o}}, \mathrm{k}_{\mathrm{H}}$, and $\mathrm{k}_{\mathrm{H}_{2}}$, represent initial bond density, $\mathrm{H}$ to $\mathrm{H}_{2}$ conversion rate, and $\mathrm{H}_{2}$ to $\mathrm{H}$ conversion rate inside $\mathrm{SiO}_{2}$ layer, respectively.

The number of interface traps that do not anneal during the relaxation phase is given by [12]:

$$
N_{I T}\left(t_{o}+t_{r}\right)=\frac{N_{I T}\left(t_{o}\right)}{1+\sqrt{\frac{\xi \cdot t_{r}}{t_{o}+t_{r}}}}
$$

where $\mathrm{N}_{\text {IT }}\left(t_{0}\right)$ is the number of interface traps at the start of the relaxation phase, $\xi$ is the diffusion coefficient during the relaxation with a value $\xi=0.5$ [12], $\mathrm{t}_{\mathrm{o}}$ is the input period and $t_{r}$ is the relaxation duration.

Interface traps at the $\mathrm{Si}-\mathrm{SiO}_{2}$ interface oppose the applied gate stress resulting in the threshold voltage increment $\left(\Delta \mathrm{V}_{\mathrm{th}}\right)$. The relation between $\mathrm{N}_{\mathrm{IT}}$ and $\Delta \mathrm{V}_{\text {th }}$ is given by:

$$
\Delta V_{t h}=(1+m) \cdot \chi \cdot q \cdot N_{I T} / C_{o x}
$$

where $\mathrm{m}, \mathrm{q}$, and $\mathrm{C}_{\mathrm{ox}}$ are the holes/mobility degradation that contribute to the $\mathrm{V}_{\text {th }}$ increment [7], electron charge, and oxide capacitance, respectively. Additionally, $\chi$ is a BTI coefficient with a value $\chi=1$ for NBTI and $\chi=0.5$ for PBTI [22].

\section{Gate Delay Analysis}

This section presents a gate delay model for BTI induced degradation and a framework for analyzing BTI in the gates.

\section{A. Gate Delay Model}

BTI induced $\Delta \mathrm{V}_{\text {th }}$ of each MOS transistor has its contribution to the additional gate delay [13]. A generalized formula that relate BTI induced $\Delta \mathrm{V}_{\mathrm{th}}$ in a transistor to the additional delay is given by [10]:

$$
\Delta D=\gamma \cdot \frac{n . \Delta V_{t h}}{\left(V_{g s}-V_{t h}\right)}
$$

where $\mathrm{n}$ is the velocity saturation index and $\gamma$ represents the stress duration with respect to the total input period (i.e., activity factor) of the transistor. The $\gamma$ dependence of the contribution to the delay reveals that transistors in a gate having different stress and relaxation phases will suffer from 
different degradations, resulting in variable contribution to the delay.

Sakuri et al. in [19] suggested that the gate output rise and fall transition times depend on the $V_{t h}$ of the PMOS and NMOS transistors, respectively. Since NBTI causes $\Delta V_{\text {th }}$ to PMOS transistor and PBTI causes $\Delta \mathrm{V}_{\text {th }}$ to NMOS transistor; therefore, analysis in this paper considers the gate rise and falling transition times for estimating NBTI and PBTI impacts, respectively. This consideration isolates BTI impacts of NMOS and PMOS transistors in a gate.

\section{B. BTI Analysis Framework}

The analysis presented in this paper addresses both NBTI and PBTI impacts in the gates. For this analysis, a framework shown in Fig. 2 has been developed. The right side of the figure is used for degradation free simulation of the gates and the left side simulate BTI impact in the gates. For degradation free case, the gates are synthesized using $45 \mathrm{~nm}$ PTM transistor models and simulated using HSPICE to get a reference for the performance metrics. Thereafter, Verilog-A modules are added to each transistor to get BTI augmented gates. Depending on biasing input of each transistor, the Verilog-A module produce $\Delta \mathrm{V}_{\mathrm{th}}$ that binds BTI impact to the additional gate delay $(\Delta \mathrm{D})$.

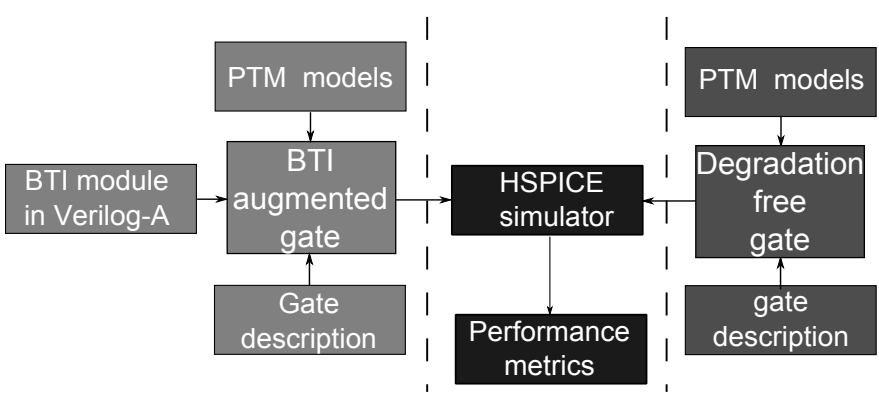

Fig. 2. Schematics of the BTI analysis framework

The framework is used for analyzing BTI impacts in MOS transistors of an inverter. It is assumed that process variations and the other failure mechanisms, e.g. Hot Carrier Degradation and Electro-migrations produce complementary additive effects so in our analysis we do not incorporate them yet. Throughout the analysis, the input has $\gamma=50 \%$ and BTI parameters used in Verilog-A module to get $\Delta \mathrm{V}_{\text {th }}$ are, $\mathrm{k}_{\mathrm{f}}=8 \times 10^{-4} \mathrm{~s}^{-1}, \mathrm{k}_{\mathrm{r}}=3 \times 10^{-18} \mathrm{~cm}^{3} \mathrm{~s}^{-1}, \mathrm{~N}_{\mathrm{o}}=5 \times 10^{16} \mathrm{~cm}^{2}$, and $\mathrm{D}_{\mathrm{H} 2}=4 \times 10^{-21} \mathrm{~cm}^{2} \mathrm{~s}^{-1}$ [11]. Fig. 3(a) gives the $\Delta \mathrm{V}_{\mathrm{th}}$ increments of MOS transistors due to BTI; it shows that $\Delta \mathrm{V}_{\text {th }}$ due to NBTI and PBTI approach $53.16 \mathrm{mV}$ and $28.08 \mathrm{mV}$, respectively, after 10 years of operation. The curves follows the $t^{1 / 6}$ trend of Eq. 1 and have a good match with the silicon results presented in $[3,4]$. The $\Delta \mathrm{V}_{\text {th }}$ is inserted into Eq. 4 to get $\Delta$ D. Fig. 3(b) shows $\Delta$ D, i.e., the percentage increment of the delay that approach $20.07 \%$ and $8.73 \%$ due to NBTI and PBTI, respectively. Therefore, it can be concluded that for a given input, (e.g., $\gamma=50 \%$ ) NBTI impact in PMOS transistors is more significant and approaches up to $2.30 \times$ the PBTI impact in NMOS transistors.

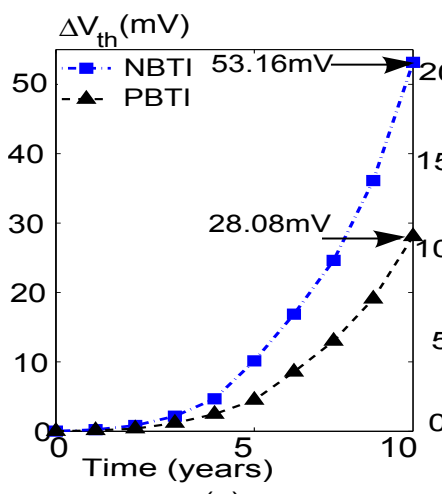

(a)

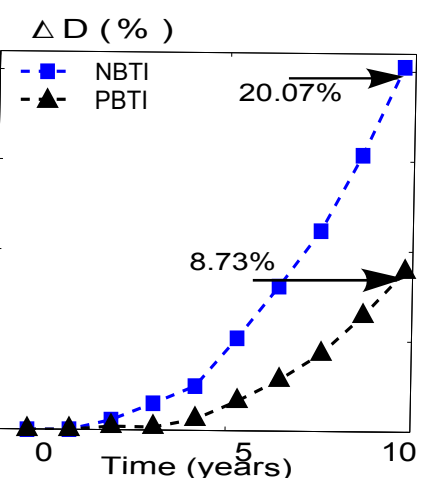

(b)
Fig. 3. (a) BTI induced $\left(\Delta \mathrm{V}_{\mathrm{th}}\right)$ increment of PMOS and NMOS transistors as a function of time (b) Inverter delays $\Delta \mathrm{D}$ increment due to NBTI and PBTI

\section{BTI IMPACTS IN BASIC GATES}

This section analyzes BTI impacts in some basic gates, such as NAND and NOR gates. Initially, it analyzes BTI dependence on transistor organizations in the gates. Thereafter, it analyzes BTI dependence on some timing parameters such as; duty cycle, frequency and stress location.

\section{A. Transistor organization Dependency}

The analysis of BTI impact presented in this paper is inspired by the observation that all MOS transistors in a gate do not contribute uniformly to the delay increment. We argue that MOS transistors connected in series have positive interference in their BTI impacts and result in a larger delay increment. On the other hand, MOS transistors connected in parallel have mutually exclusive BTI impacts that result in smaller delay increment. To justify the argument, two basic gates -NAND and NOR- are analyzed for $\gamma=50 \%$ at the inputs.

\section{NAND gate}

Let us consider a two inputs NAND gate shown in Fig. 4(a). The gate consists of two NMOS transistors $\left(\mathrm{N}_{1}\right.$ and

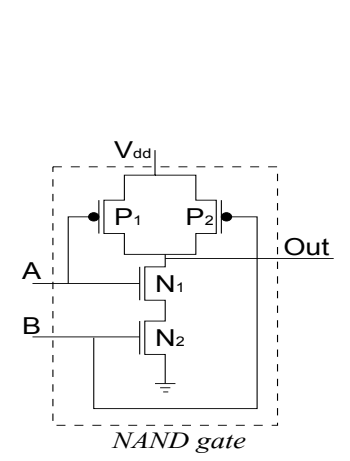

(a)

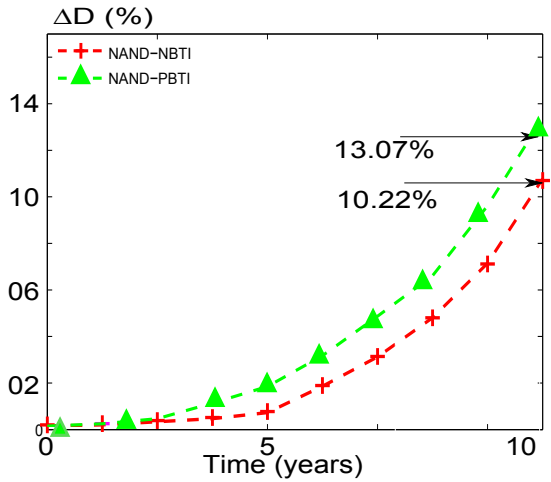

(b)
Fig. 4. (a) Transistor organization in a NAND gate (b) Percent delays of NAND gate due to NBTI and PBTI 


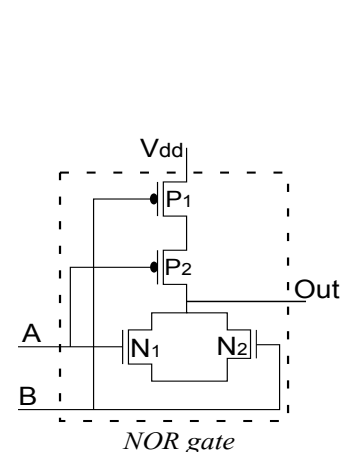

(a)

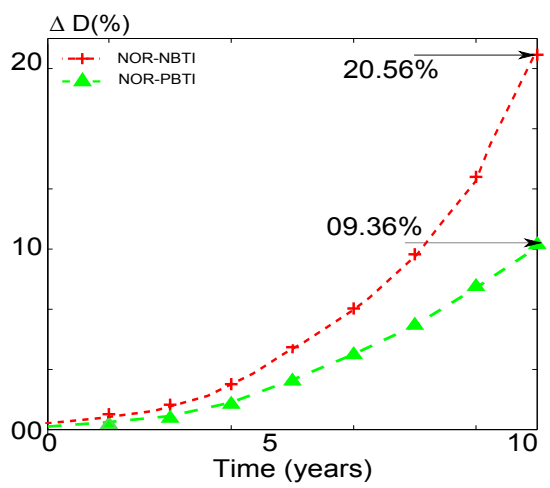

(b)
Fig. 5. (a) Transistor organization in a NOR gate (b) Percent delays of NOR gate due to NBTI and PBTI

$\mathrm{N}_{2}$ ) connected in series, as well as two PMOS transistors $\left(\mathrm{P}_{1}\right.$ and $\mathrm{P}_{2}$ ) connected in parallel. The gate is analyzed using the framework mentioned in the previous section; the simulation results are shown in Fig. 4(b). Analysis of the results reveal that PBTI impact on the serially connected NMOS transistors agglomerates and causes about $13.07 \%$ additional delay. On the other hand, NBTI impact in the parallel connected PMOS transistors have mutually exclusive impacts and cause only up to $10.22 \%$ additional delay.

Therefore, it can be concluded that PBTI impact dominates in NAND gate and is up to $1.27 \times$ the NBTI impact.

\section{NOR gate}

To strengthen the claim of BTI dependence on transistor organization, a two inputs NOR gate shown in Fig. 5(a) is considered. The gate consists of two serially connected PMOS transistors $\left(\mathrm{P}_{1}\right.$ and $\left.\mathrm{P}_{2}\right)$ as well as two parallel connected NMOS transistors $\left(\mathrm{N}_{1}\right.$ and $\left.\mathrm{N}_{2}\right)$. The gate is analyzed using the framework and the results are shown in Fig. 5(b). It shows that NBTI impact in the serially connected PMOS transistors amasses to cause up to $20.56 \%$ additional delay, while PBTI impact in the parallel connected transistors can only cause 9.36\% additional delay.

Therefore, it can be concluded that NBTI impact dominates in NOR gate and is up to $2.19 \times$ higher than the PBTI impact. We can conclude that opposite effects are present for NBTI/PBTI ratio depending on the transistor topology.

\section{B. Timing Dependency}

In addition to the dependency on transistor organization inside a gate, BTI has a strong dependence on the gate inputs. This section presents the impacts of three input parameters such as; duty cycle, stress location and frequency on BTI.

\section{Duty cycle dependence}

Duty cycle is defined as the percentage of a period that the inputs of a gate remains high. Note that high and low inputs cause stress and recovery phases to the NMOS transistors, respectively; it cause reverse phases to the PMOS transistors. Since stress and relaxation phases have symmetric processes

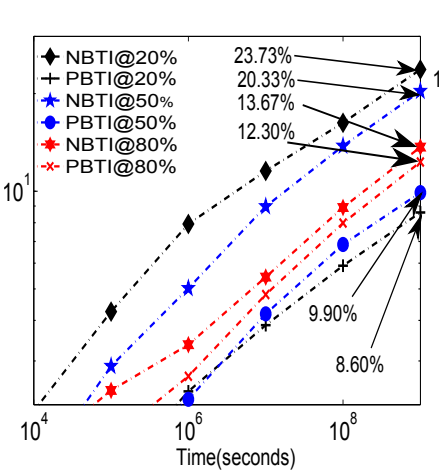

(a)

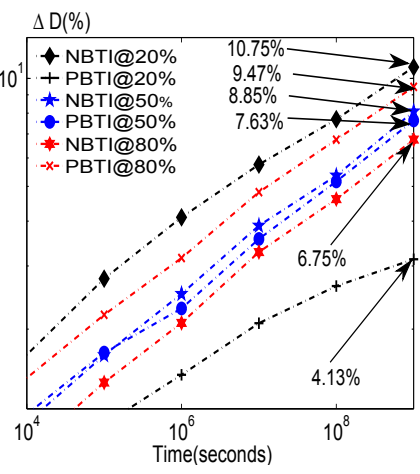

(b)
Fig. 6. (a) Percent delay increments of NOR due to NBTI and PBTI at $20 \%$, $50 \%$ and $80 \%$ input duty cycles (b) Percent delay increments of NAND due to NBTI and PBTI at $20 \%, 50 \%$ and $80 \%$ input duty cycles

(i.e., $\equiv \mathrm{Si}-\mathrm{H}$ bond breaking and recovery), the dominance of one over the other is determined by stress and relaxation phase durations.

The two basic gates -NOR and NAND- are analyzed under three duty cycles $-20 \%, 50 \%$, and $80 \%$ - to evaluate the BTI impact. Fig. 6(a) shows the BTI induced delay increments in NOR gate under the three duty cycles. The figure shows that variation in the duty cycle has a significant impact on the BTI induced delays. For example, at $50 \%$ duty cycle in NOR gate, NBTI and PBTI cause $20.33 \%$ and $9.90 \%$ additional delays, respectively. However, at $20 \%$ duty cycle, the additional delay due to NBTI increases to $23.73 \%$, which is $1.20 \times$ the $50 \%$ duty cycle case; while the delay due to PBTI becomes only $8.60 \%$, which is $0.85 \times$ the $50 \%$ duty cycle case. However, it is observed that at $80 \%$ duty cycle, NBTI brings only $13.67 \%$ additional delay while PBTI causes $12.30 \%$ additinal delay. Similar, analyses are carried for a NAND gate and results are shown in Fig. 6(b).

It can be concluded, at lower duty cycles, NBTI is more significant than PBTI on the gates. However, higher duty cycles magnify PBTI and diminish NBTI impacts on the gates.

\section{Frequency Dependence}

Currently, there is no clear explanation of BTI dependence on frequency at the transistor level. Alam et al., in [5] have claimed frequency independence due to equivalent number of $\mathrm{Si}-\mathrm{H}$ bond breaking and annealing during stress and relaxation phases, respectively. While Tibor et al., in [9] suggested weak frequency due to asymmetry between the bonds breaking and recovery during stress-recovery phases . Fig. 7(a), based on the model presented in Eq. 1 and Eq. 2, shows the BTI analysis of NAND gate at different frequencies (i.e., 333.33, 166.66 and $83.33 \mathrm{MHz}$ ) but identical duty cycles. The figure shows that the BTI -both NBTI and PBTI- induced delays are close the nominal values even with $4 \times$ variation in the frequency. Fig. 7(b) shows similar observation for a NOR gate. The figure shows that $4 \times$ variation in frequency brings negligible variation to the NBTI and PBTI impacts on NOR gate. 


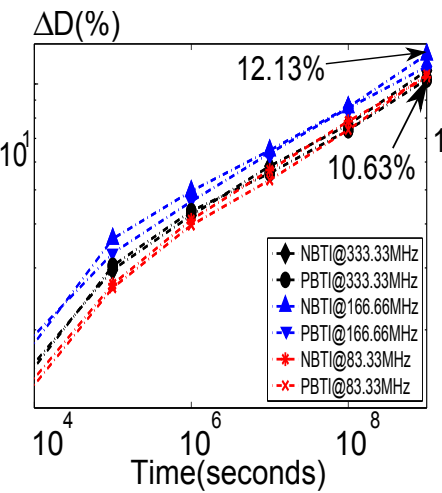

(a)

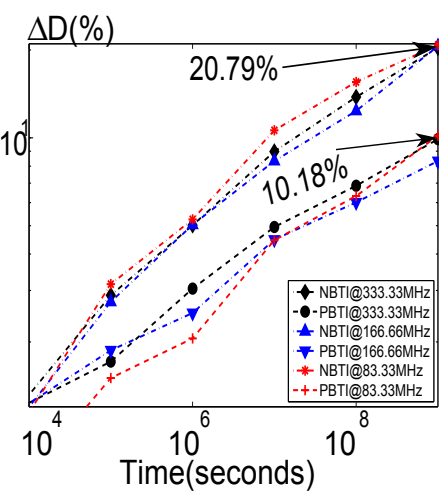

(b)
Fig. 7. (a) NBTI and PBTI induced delays in a NAND gate at different frequencies and identical duty cycles (b) NBTI and PBTI induced delays in a NOR gate at different frequencies and identical duty cycles

Therefore, it can be deduced that BTI is independent of the operating frequency. The analysis is consistent with the claim of frequency independence presented in [5].

\section{Stress Location Dependence}

Majority of the published literature estimated BTI impact with the assumption that a given $\Delta \mathrm{V}_{\text {th }}$ in any transistor contribute uniformly to delay increment of the gate. However, in reality, the contribution to the delay increment depends on the location of the stressed transistor. We claim that for a given $\Delta \mathrm{V}_{\text {th }}$ in a transistor closer to $\mathrm{V}_{\mathrm{dd}}$, the contribution to the delay increment is lower. Conversely, the same $\Delta V_{\text {th }}$ in a transistor closer the ground has higher contribution to the delay increment.

The stress location dependency is analyzed in the two basic gates. Fig. 8(a) shows the delay increment due to PBTI induced $\Delta V_{\text {th }}$ in $\mathrm{N}_{1}$ and $\mathrm{N}_{2}$ of Fig. 4(a). The figure shows that $\Delta V_{\text {th }}$ in $\mathrm{N}_{1}$ causes $3.10 \%$ to the gate. However, the same $\Delta \mathrm{V}_{\text {th }}$ in $\mathrm{N}_{2}$ causes $5.62 \%$ delay increment. The higher contribution to the delay increment can be contributed to the lower driving potential at the source of $\mathrm{N}_{2}$ due to drop in $\mathrm{N}_{1}$. Similarly, Fig. 8(b) shows the difference in delay increment due to NBTI induced $\Delta V_{\text {th }}$ in $\mathrm{P}_{1}$ and $\mathrm{P}_{2}$ of Fig. 5(a); which

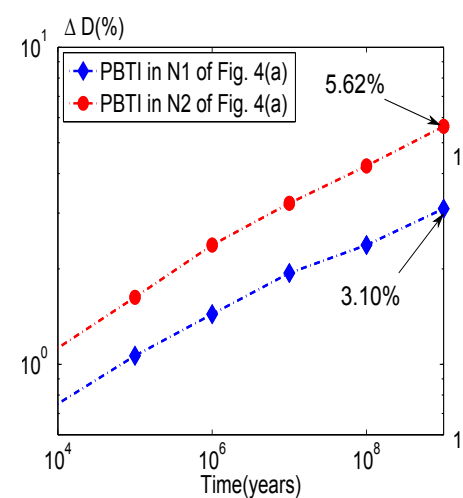

(a)

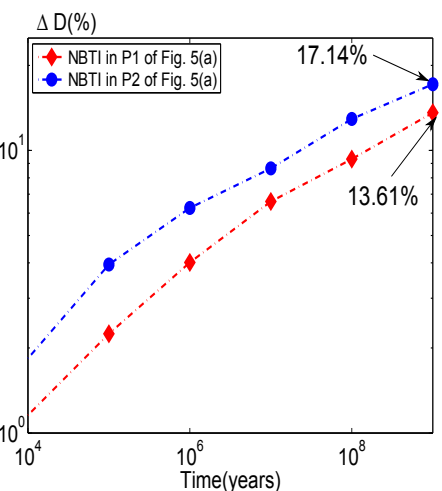

(b)
Fig. 8. (a) Percent delay increment of NAND gate due to PBTI induced $\Delta \mathrm{V}_{\text {th }}$ in transistor $\mathrm{N}_{1}$ and $\mathrm{N}_{2}$ of Fig. 4a (b) Percent delay increment of NOR gate due to NBTI induced $\Delta V_{\text {th }}$ in transistor $P_{1}$ and $P_{2}$ of Fig. 5a shows that NBTI in $\mathrm{P}_{2}$ causes $17.14 \%$ delay increment, while NBTI in $\mathrm{P}_{1}$ causes only $13.61 \%$ delay increment.

\section{BTI IMPACTS IN COMPLEX GATES}

This section analyzes BTI impacts in the complex gates. Initially, it explores BTI in some complex gates and then investigates its timing dependencies.

\section{A. Transistor Organization Dependency}

Complex gates realize complex logic functions using suitable MOS transistors configuration. Due to these transistors configurations, exact estimation of BTI impacts in complex gates is more challenging.

As an example case, the logic function $(\overline{A .(B+C)+D . E})$ is implemented by the transistor configuration show in Fig. 9(a). The gate has at most three serially connected PMOS transistors $\left(\mathrm{P}_{2}, \mathrm{P}_{3}, \mathrm{P}_{5}\right)$ and two NMOS transistors $\left(\mathrm{N}_{1}, \mathrm{~N}_{2}\right.$ or $\mathrm{N}_{4}, \mathrm{~N}_{5}$ ). The gate is analyzed using the framework of Section III-A and the results are shown in Fig. 9(b). The figure reveals that under the worst case $-\mathrm{P}_{2}, \mathrm{P}_{3}, \mathrm{P}_{5^{-}}$are under stress, NBTI impact $-21.56 \%$ additional delay- is about $2.85 \times$ the PBTI impact $-7.59 \%$ additional delay-. The higher difference in the impacts can be attributed to the accumulative nature of the NBTI impact on the three serially connected PMOS transistors.

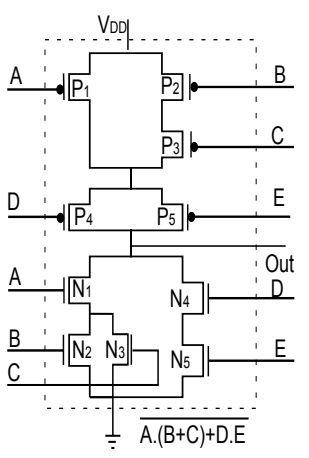

(a)

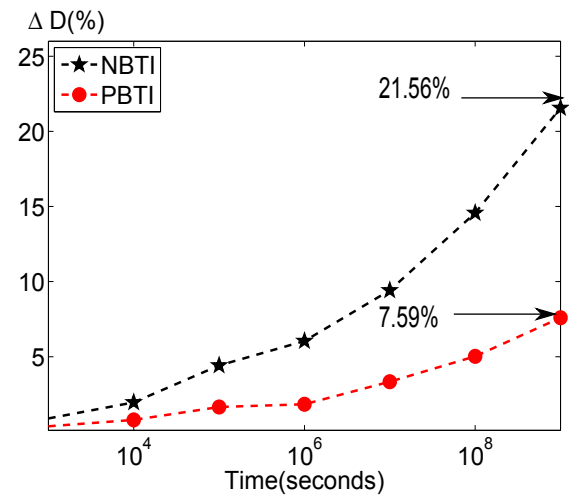

(b)
Fig. 9. (a) Transistor organization in a complex gate implementing $\overline{A .(B+C)+D \cdot E}$ function (b) Percent delay (rising and falling output transition time increment) of the complex gate due to NBTI and PBTI

Moreover, to extend BTI analysis in other complex gates. Table 1 shows analysis of two well known complex gates i.e. And-Or-Invert (AOI) and Or-And-Invert (OAI). The table

TABLE I

NBTI AND PBTI IMPACTS IN DIFFERENT GATES

\begin{tabular}{|c|c|c|c|c|}
\hline Gate & Max. PMOS in series & Min. NMOS in series & $\Delta \mathrm{D}_{\text {NBTI }}(\%)$ & $\Delta \mathrm{D}_{\mathrm{PBTI}}(\%)$ \\
\hline AOI & 3 & 1 & 23.60 & 7.61 \\
\hline OAI & 3 & 2 & 21.24 & 13.76 \\
\hline
\end{tabular}

$\mathrm{AOI}=\mathrm{And}$ Or Invert, $\mathrm{OAI}=$ Or And Invert

shows that depending on the number of serially or parallel connected PMOS/NMOS transistors, the impact of NBTI/PBTI becomes more significant. We can conclude that series and parallel connected transistors determine the dominant impact. Additionally, the sizes need to be differently selected to enable delay balancing, and that influence the NBTI/PBTI impacts. 


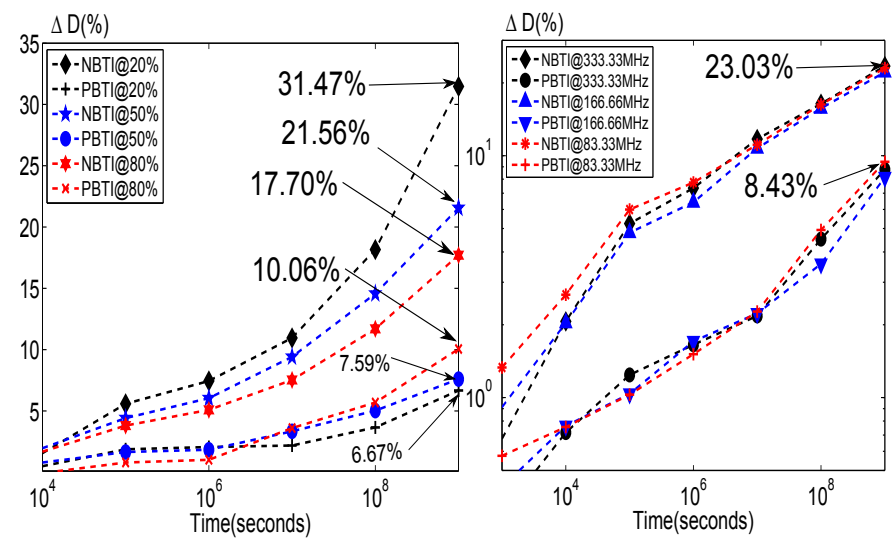

Fig. 10. (a) Percent delay increments in the complex gate of Fig.9(a) at different duty cycles (b) Percent delay increments in the complex gate of Fig.9(a) at different frequencies

\section{B. Timing dependency}

Impacts of the three timing parameters are analyzed in the complex gate (see Fig. 9(a)). For duty cycle variation analysis, Fig 10(a) shows that NBTI induced delay increment reaches to $31.47 \%$ at $20 \%$ duty cycle. The increment is $45 \%$ higher than the 50\% duty cycle case. Fig 10(b) shows BTI induced delay increment with the frequency variation and confirms our previous claim of the frequency independence.

The stress location dependency in pull-up network complex gate implementing $\overline{A . B+C . D+E}$, shown in Fig. 11(a) is analyzed. Initially, NBTI is inserted in $\mathrm{P}_{1}$ and $\mathrm{P}_{2}$ and all the other PMOS transistors are degradation free. Simulation results under this condition is shown in Fig. 11(a), which shows that NBTI causes $6.41 \%$ delay increment. However, when NBTI only effects $\mathrm{P}_{5}$ and all the other transistors are degradation free, the delay incremet reaches $14.75 \%$. The $2.28 \times$ higher impact can be attributed to lower source voltage of $\mathrm{P}_{5}$ due voltage drop in the upper PMOS transistors.

\section{CONCLUSION}

This paper presented a BTI analysis in both basic and complex gates. First, the transistor level analysis revealed that NBTI causes $2.30 \times$ more degradation to the PMOS transistors than PBTI causes to the NMOS transistors. Second,

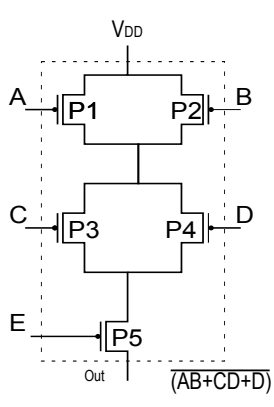

(a)

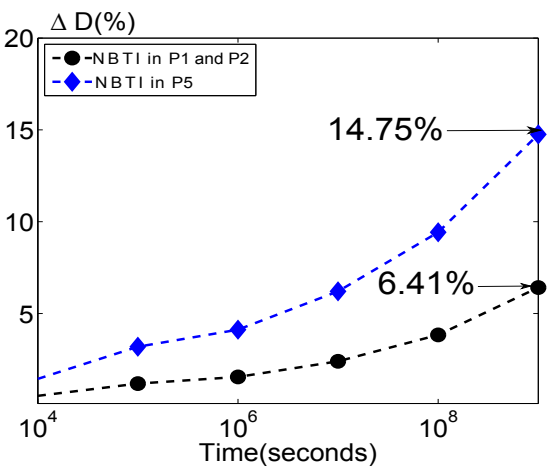

(b)
Fig. 11. (a) Transistor organization in pull-up network of complex gate implementing $\overline{A . B+C . D+E}$ function (b) Percent increment in rising transition time due to NBTI considering transistor organization in gates showed that NBTI impact is more significant in NOR gates and PBTI brings more degradation to the NAND gates. Third, inputs timing dependencies of BTI showed; (a) input duty cycle has a significant impact on NBTI or PBTI dominance in a gate,(b) BTI is frequency independent, and (c) location of the stressed transistor causes a significant variation to the BTI impact on the gates.

\section{REFERENCES}

[1] S. Borkar, et al "Micro architecture and Design Challenges for Giga scale Integration", Pro. of Intl. Sympos. Micro architecture, 2004.

[2] N. Kizmuka "The Impact of BTI for Direct Tunneling Ultra Thin Gate Oxide of MOSFET Scaling", VLSI Technology, Digest of Technical Papers, pp: 73-74, 1999.

[3] S. Zafar, et al., "A comparative study of NBTI and PBTI in $\mathrm{SiO} 2 / \mathrm{HfO} 2$ stacks with FUSI, TiN, gates", Pro. of VLSI Technology symp., 2006.

[4] W. Abadeer, et al., "Behaviour of NBTI Under AC Dynamic Circuit Conditions", Pro. of Intl. Physics Reliability Symp., pp: 17- 22, 2003.

[5] M.A. Alam, et al., "A Comprehensive Model of PMOS NBTI Degradation", Microelectronics Reliability, Vol:45, Issue:1, pp: 71-81, 2005.

[6] B. Kaczer, et al., "Disorder-Controlled-Kinetics Model NBTI and its Experimental Verification", Proc. Intl. Physics Reliability Symp., 2005.

[7] A.T. Krishnan, et al., "NBTI impact on transistor and circuit: Models, mechanisms and scaling effects", Pro. of IEDM, 2003.

[8] A.E. Islam, et al., "On the possibility of degradation-free field effect transistors", Applied Physics Letter, 2008.

[9] T. Grassor, et al., "Critical Modeling Issues in Negative Bias Temperature Instability", 215 th ECS meeting, pp: 265-287, 2009.

[10] B.C. Paul, et al., "Impact of NBTI on the Temporal Performance Degradation of Digital Circuits", IEEE Electron Device Letter, Vol. 26, No.8, Aug. 2005.

[11] H. Kufluoglu, et al., "A Generalized Reaction Diffusion Model With Explicit H-H2 Dynamics for Negative Bias Temperature Instability (NBTI) Degradation", IEEE Transaction of Elect. Devices, 2007.

[12] S.V. Kumar, et al., "An Analytical Model for Negative Bias Temperature Instability ", Proc. of ICCAD, 2006.

[13] S. Khan, et al., "Temperature dependence of NBTI induced delay", Proc. of Intl. Online Test Symp. (IOLTS), Page: 15-20, July, 2010.

[14] H. Luo, et al., "A Novel Gate-Level NBTI Delay Degradation Model with Stacking Effect" Springer Lecture Notes in Computer Science, pp: 160-170, 2007.

[15] R. Vattikonda, et al., "Modeling and minimization of PMOS NBTI effect for robust nanometer design", Proc. of Design Automation Conference (DAC), pp:1047 - 1052, 2006.

[16] S. Khan, et al., "NBTI Monitoring and Design for Reliability in Nanoscale Circuits ", proc. of IEEE Sym. on Defect and Fault Tolerant Systems, pp. 68-76, Oct. 2011.

[17] W. Wang, et al., "NBTI Impact on Combinational and Sequential Circuits“, Proc. of Design Automation Conference, pp. 546-551, 2007.

[18] A.T. Krishnan, et al., "NBTI impact on transistor and circuit: models, mechanisms and scaling effects ", Proc. of Intl. Electronics Device Meeting(IEDM), pp:14.5.1 - 14.5.4, 2003.

[19] T. Sakurai, et al., "Alpha-Power law MOSFET model and its applications to CMOS delay and other formulas", IEEE J. Solid-State Circuits, Vol.25, No.2, April 1990,

[20] H. Wang, et al., "Systematic Analysis of Energy and Delay Impact of Very Deep Sub-micron Process Variability Effects in Embedded SRAM Modules" Proc. of DATE., pp: 914 - 919, 2005.

[21] B. Kaczer, et al., "Time and Workload Dependent Device Variability in Circuit Simulations“ Proc. Intl. Conf on IC Design and Technology, pp: $1-4,2011$.

[22] M.T. Luque, et al., "From Mean Values to Distribution of BT Lifetime of Deeply scaled FETs through Atomistic Understanding of the Degradation“ Sym. on VLSI Technology, pp: 152-153, 2011.

[23] Predictive Technology Model "http://ptm.asu.edu/", 